\title{
Free Volume, Molecular Mobility and Polymer Structure: Towards the Rational Design of Multi-Functional Materials
}

\author{
M. Roussenova ${ }^{a}$, D.J. $\operatorname{Hughes}^{a}$, J. Enrione ${ }^{b}$, P. Diaz-Calderon ${ }^{b}$, E. Sivaniah $^{c}$,

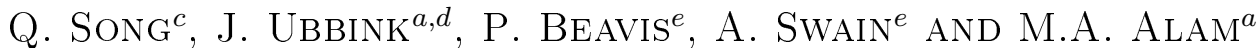 \\ ${ }^{a}$ H.H. Wills Physics Laboratory, University of Bristol, Tyndall Avenue, Bristol BS8 1TL, UK \\ ${ }^{b}$ Department of Food Science and Technology, Universidad de Santiago de Chile \\ Av. Ecuador 3343, Estación Central, Santiago, Chile \\ ${ }^{c}$ University of Cambridge, Cavendish Laboratory, Biological and Soft Systems Section, Cambridge CB3 0HE, UK \\ ${ }^{d}$ Food Concept \& Physical Design "The Mill", Mühleweg 10, CH-4112 Flüh, Switzerland \\ ${ }^{e}$ AWE, Aldermaston, Reading, RG7 4PR, UK
}

\begin{abstract}
In this short paper we illustrate the versatility of positron annihilation lifetime spectrometry when used for material characterisation by presenting a selection of recent lifetime studies carried out on novel polymeric materials with a wide range of potential industrial applications. We highlight the importance of local free volume in governing the macroscopic properties (e.g. permeation properties, mechanical strength) of these materials and how lifetime measurements can be used in conjunction with a number of complementary techniques in order to establish composition-structure-property relationships for these systems.
\end{abstract}

DOI: $10.12693 /$ APhysPolA.125.801

PACS: 78.70.Bj, 82.35.Pq, 66.30.je, 82.35.Np, 81.05.Qk

\section{Introduction}

Over the past decades the development of advanced multifunctional polymeric materials with tunable chemical functionalities, exceptional physical and mechanical properties has become a subject of great scientific interest, not only from a fundamental perspective but also due to their broad potential industrial applications [1-3]. Despite the strong interest, however, to date most of these polymeric materials are developed using mainly trial-and-error strategies, making the rational control of their physical properties a very difficult task. For this reason, in recent years alternative approaches, which establish a fundamental understanding of the materials at a molecular level and employ composition-structure-property relationships to predict and rationally control their physical and mechanical properties, have become increasingly more popular.

It is now well known that both the structure and dynamics of polymeric materials are closely related to the local free volume which exists between molecules in these systems due to irregular packing, density fluctuations and topological constraints [4]. The concept of free volume is important, since it is commonly considered as the volume enabling molecular re-organisation and may, therefore, be associated with phenomena such as diffusion, viscoelastic flow and the glass transition [4-7]. Positron annihilation lifetime spectrometry (PALS) is a well established technique which can probe this local free volume directly,

*corresponding author; e-mail: m.roussenova@bristol.ac.uk thus providing an invaluable tool for studying the structure and dynamics of polymeric materials [8,9]. Furthermore, by using PALS in conjunction with a number of complementary techniques, the changes in local free volume observed at the molecular length scale may be related to the changes in the macroscopic bulk properties (e.g. density) [10-12]. This renders an integrated approach for establishing composition-structure-property relationships which provide a stepping stone to the rational control of the material properties at the molecular level.

\section{PALS: A free volume perspective}

In this brief overview, we illustrate the versatility of PALS in this respect, by presenting a selection of recent studies carried out in Bristol which focus on the rational design of novel polymeric materials with a number of different industrial applications. The examples selected aim to elucidate the importance of local free volume for range of different phenomena, for example, molecular mobility, diffusion/permeation, thermodynamic state and mechanical strength.

\subsection{Zeolitic imidazolate framework (ZIF-8)-polyimide composite membranes for gas separation: permeability and local free volume}

The development of next-generation polymer-composite membranes for gas separation has attracted significant interest in recent years, due to the fact that conventional polymer membranes suffer from a trade-off between gas permeability and selectivity, i.e. polymers with high permeability have low selectivity and 
vice versa [13]. One promising approach has been the preparation of mixed matrix membranes incorporating zeolitic imidazolate frameworks (ZIFs), a sub-family of metal-organic frameworks with diverse framework architectures, functional properties and tunable pore sizes [14]. ZIF-based membranes have previously shown molecular sieving effect with high permeability, without significant loss in selectivity [15]. However, the mechanism of gas transport in these materials has not yet been elucidated.

Here, we illustrate how PALS can be used in conjunction with gas permeation tests (utilising the constant-volume variable pressure method and $\mathrm{H}_{2}, \mathrm{CO}_{2}$, and $\mathrm{O}_{2}$ as test gases [15]), in order to gain a better understanding of the gas transport properties of novel Matrimid 5218 (polyimide, Huntsman Advanced Materials)-ZIF-8 composite membranes. Matrimid based membranes incorporating different amounts of well dispersed ZIF-8 nanocrystals ( $0-30 \mathrm{wt} \%$ ) were prepared and their physico-chemical characteristics are summarised in Table I. Full details on the synthesis of these membranes and the experimental methods can be found in Ref. [15].

TABLE I

Physico-chemical characteristics and lifetime parameters measured for the Matrimid-ZIF-8 membranes. Reproduced from Ref. [15] with permission from the Royal Society of Chemistry.

\begin{tabular}{c|cc|cccc}
\hline \hline $\begin{array}{c}Q_{\mathrm{ZIF}-8} \\
{[\mathrm{wt} \%]}\end{array}$ & $\begin{array}{c}T_{\mathrm{g}} \\
{[\mathrm{K}]}\end{array}$ & $\begin{array}{c}\rho \\
{\left[\mathrm{g} \mathrm{cm}^{-3}\right]}\end{array}$ & $\begin{array}{c}\tau_{\mathrm{o}-\mathrm{Ps}, 1} \\
{[\mathrm{~ns}]}\end{array}$ & $\begin{array}{c}I_{\mathrm{o}-\mathrm{Ps}, 1} \\
{[\%]}\end{array}$ & $\begin{array}{c}\tau_{\mathrm{o}-\mathrm{Ps}, 2} \\
{[\mathrm{~ns}]}\end{array}$ & $\begin{array}{c}I_{\mathrm{o}-\mathrm{Ps}, 2} \\
{[\%]}\end{array}$ \\
\hline 0 & 330 & 1.20 & $1.19 \pm 0.06$ & $1.8 \pm 0.3$ & $4.62 \pm 0.08$ & $2.3 \pm 0.1$ \\
5 & 336 & 1.19 & $1.53 \pm 0.10$ & $2.0 \pm 0.1$ & $4.99 \pm 0.20$ & $2.4 \pm 0.1$ \\
10 & 346 & 1.18 & $1.43 \pm 0.07$ & $2.3 \pm 0.1$ & $4.77 \pm 0.18$ & $2.7 \pm 0.1$ \\
20 & 343 & 1.15 & $1.54 \pm 0.05$ & $3.1 \pm 0.1$ & $4.75 \pm 0.23$ & $3.0 \pm 0.2$ \\
30 & 349 & 1.13 & $1.67 \pm 0.09$ & $3.2 \pm 0.1$ & $5.24 \pm 0.12$ & $4.4 \pm 0.2$
\end{tabular}

It can be seen from Table I that the addition of ZIF-8 nanoparticles causes a systematic increase in the glass transition temperature, $T_{\mathrm{g}}$, of the polyimide based membranes, in agreement with previous observations reported for similar systems [15].

In Fig. 1 we present the gas transport data for the polyimide-based membranes with various compositions, annealed under vacuum at $\approx 500 \mathrm{~K}$ for $18 \mathrm{~h}$ (in order to remove any residual solvent). In Fig. 1a we observe a molecular sieving effect with the gas permeability following the kinetic diameter of the respective gas molecules: $\mathrm{H}_{2}(2.89 \AA)>\mathrm{CO}_{2}(3.3 \AA)>\mathrm{O}_{2}(3.46 \AA)$ [16]. Furthermore, the permeability of all gases increases as a function of increasing ZIF-8 content and the effect is most pronounced in the case of $\mathrm{H}_{2}$, where we measure a $250 \%$ increase in permeability over the ZIF- 8 content range studied. It can also be seen from Fig. $1 \mathrm{~b}$ that the selectivity $\left(\alpha_{A / B}=P_{A} / P_{\mathrm{B}}\right.$, where $P_{A}$ and $P_{\mathrm{B}}$ are the permeabilities of the two respective gases) remains largely unchanged compared with the pure Matrimid membrane, highlighting the potential for industrial application. In Table I we also report the lifetime measurements for the Matrimid-based composite membranes. Two distinct o-Ps lifetimes can be resolved in the spectra measured for the membranes, suggesting they have a bimodal free volume distribution [17]. The ZIF-8 nanoparticles possess pores with a diameter of $11.6 \AA$, connected by smaller apertures, $3.4 \AA$ in diameter [18].
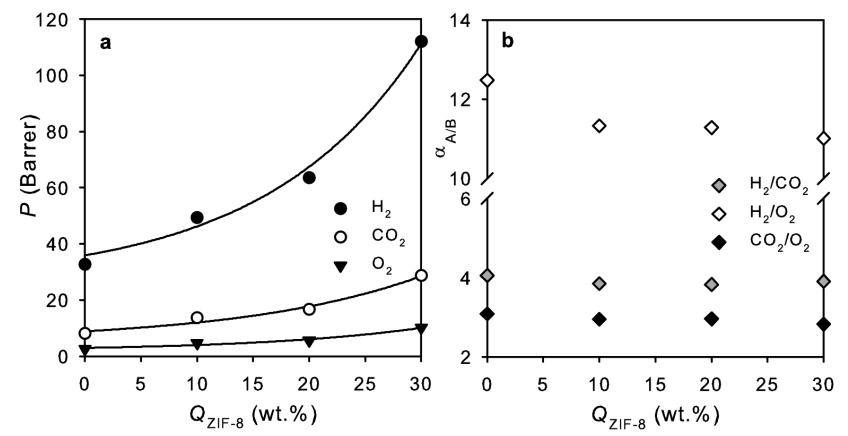

Fig. 1. Pure gas permeation properties: (a) permeability and (b) selectivity of the Matrimid-based membranes with different ZIF-8 loading levels [15]. The solid lines are included as guides to the eye. Adapted from Ref. [15] with permission from the Royal Society of Chemistry.

One would expect the lifetimes of $o$-Ps annihilating within these pores to be of the order of $5 \mathrm{~ns}$ and $1 \mathrm{~ns}$, respectively [9]. These lifetimes are too similar to the two $o$-Ps lifetimes measured in the neat Matrimid membrane, meaning that they cannot be resolved as separate components in the spectra of the composite membranes. This suggests that the reported lifetimes reflect the changes in molecular packing of the base Matrimid polymer [15]. It is clear from Table I that although the changes in the longer o-Ps component are not very pronounced, there is a systematic increase in the shorter $o$-Ps lifetime with increasing ZIF-8 content (with the exception of 5 wt\% ZIF-8 membrane). This suggests that the ZIF-8 inclusions alter the molecular packing of the polyimide based membranes, leading to an increase in the average size of the local free volume elements. The effect of the ZIF-8 nanoparticles on the permeation properties of the membranes is, therefore, twofold: they loosen the molecular packing of the base polymer (accompanied by a decrease in density, as shown in Table I), while allowing the gas molecules to freely diffuse through their porous structure. Both of these effects lead to improved permeability compared to the neat Matrimid membrane [15].

\subsection{Biopolymer based pharmaceutical encapsulants: molecular mobility, thermodynamic state and local free volume}

Biopolymers such as gelatin are widely used in the pharmaceutical industry for the formulation of excipients and encapsulation matrices for labile bioactive ingredients [2]. Gelatin is particularly useful for this purpose due to its thermally reversible gelation close to the human body temperature, as well as its ability to produce films which can readily dissolve in water $[2,3]$. However, alongside the large number of useful properties inherent to gelatin, it also exhibits some significant shortcomings, 
for example, it is highly brittle at low water contents which may result in the undesired failure of glassy gelatin encapsulants. In order to overcome this problem, gelatin matrices are often modified by the inclusion of polyol molecules such as glycerol and sorbitol [3]. Both glycerol and sorbitol have been previously shown to improve the mechanical properties of glassy gelatin films, as illustrated in the inset of Fig. 2a which shows a significant reduction in the force at breaking point, $F_{\mathrm{B}}$, as a function of increasing polyol content [19]. It has also been previously shown that the permeability of gelatin films towards gases and water vapour may also be altered by the addition of such low molecular weight additives $[3,20]$.
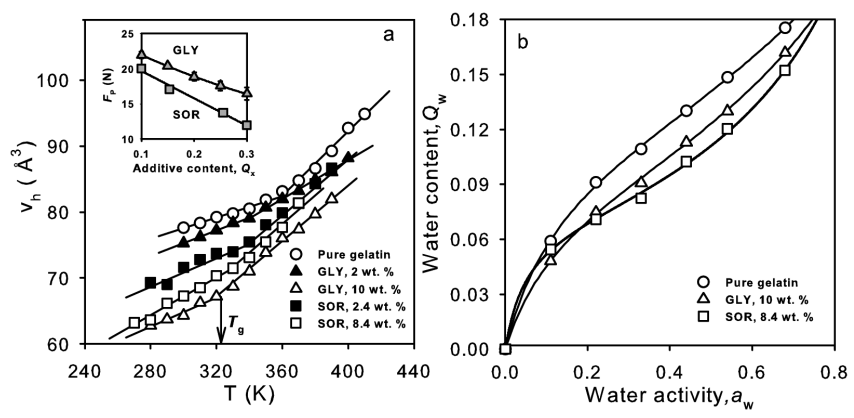

Fig. 2. (a) Temperature dependent lifetime measurements for gelatin oligomer matrices with different compositions equilibrated at $a_{\mathrm{w}}=0.11$. Inset: Systematic decrease in the force at breaking point, $F_{\mathrm{B}}$ (determined by puncture tests, as described in Ref. [19]) of gelatin oligomer films as a function of increasing polyol content. Adapted from Ref. [19]. Copyright (2001) Elsevier. (b) Water vapour sorption isotherms measured at $T=298 \mathrm{~K}$ for gelatin oligomers with different compositions [20].

It is commonly invoked that the molecular mobility, and hence the barrier properties of biopolymer matrices are related to the local free volume $[5,7]$. However, to date there is limited information about the nature of this free volume in gelatin oligomers and how it is affected by factors such as temperature, matrix composition and water content. Here, we present a selection of lifetime measurements in order to elucidate the effects of temperature and low molecular weight diluents (namely water, glycerol and sorbitol) on the thermodynamic state and local free volume of low water content bovine gelatin matrices. We also aim to highlight the versatility of PALS when used for the study of biopolymers and how it can be used in conjunction with complementary techniques to establish composition-structure relationships for such systems. Details on the preparation of the gelatin-polyol matrices and the experimental methods can be found in Ref. [20].

It can be seen from Fig. $2 \mathrm{a}$ that the average hole size of the gelatin oligomer matrices shows a strong temperature dependence with a clear change in gradient at the glass transition temperature, $T_{\mathrm{g}}$. The $T_{\mathrm{g}}$ values derived from the PALS measurements are in excellent agreement with the complementary values obtained by differential

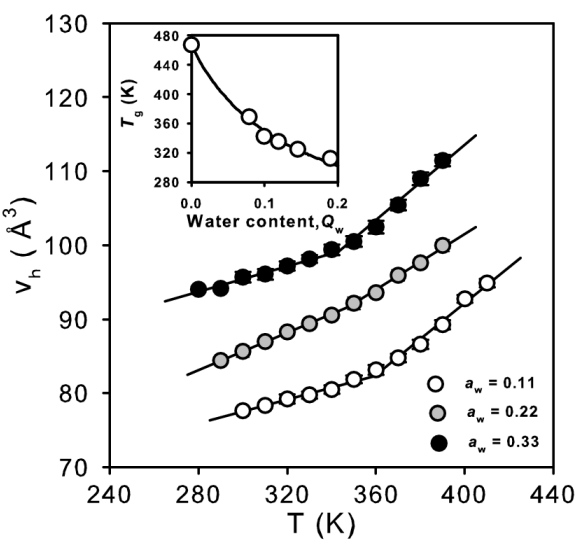

Fig. 3. Temperature dependent lifetime measurements for pure gelatin oligomers equilibrated at different water activities, $a_{\mathrm{w}}$. Inset: Decrease in the glass transition temperature, $T_{\mathrm{g}}$, of the gelatin oligomer matrices as a function of increasing water content [20].

scanning calorimetry (data not shown) [20]. It is also clear that both, glycerol and sorbitol act as packing enhancers, causing a reduction in the average hole size of the gelatin oligomer matrices (both in the glassy and rubbery states), accompanied by a reduction in $T_{\mathrm{g}}$. Furthermore, as shown in Fig. 2b, both polyols effectively alter the water sorption behaviour of the gelatin matrices, causing a decrease in the absorption of water vapour at well defined water activities [20]. For example, at $a_{\mathrm{w}}=0.33$ we observe $\approx 25 \%$ decrease in water content upon the addition of $8.4 \mathrm{wt} \%$ sorbitol. This is advantageous from a practical point of view since water is a highly efficient plasticiser for biopolymer matrices $[3,10]$. It has a profound effect on their thermodynamic state and physical structure, as illustrated in Fig. 3. Water causes a strong depression of the glass transition temperature of the gelatin oligomer matrices (inset) which is accompanied by a significant increase in the average hole size of the matrices (both in the glassy and rubbery states) [10-12, 21, 22]. In addition, plasticisation is commonly accompanied by a significant increase in the diffusional mobility of small molecules (e.g.: gases and volatile organic compounds), which can lead to a deterioration of the encapsulation efficiency of the gelatin matrices [3].

\subsection{Novel poly(dimethylsiloxane) based polymer-nanoparticle composites: mechanical reinforcement and local free volume}

Investigations into the effect on macroscopic properties of polymer matrices incorporating nanoscale fillers have become popular in recent years [23]. The physical, chemical, and mechanical improvements in such systems have been found to be significantly greater than more traditional polymer-composites where micron sized inclusions are commonplace. The origin of these macroscopic property improvements is still unclear, believed to be as a result of enhanced polymer-filler interactions occurring at a 
molecular level. With the presence of enhanced polymer-particle interactions in polymer-nanoparticle composites [24], it is hypothesised that these enhancements may manifest themselves through changes in the local free volume, measureable by PALS, giving insight into the mechanism of macroscopic mechanical reinforcement from changes at a molecular level.

In this study the effect of incorporating, through physical dispersion, various concentrations (expressed as wt\%) of meta-carborane $(m-\mathrm{CB})$ nanoparticles into a monomodal cross-linked poly (dimethylsiloxane) (PDMS) network (detailed sample information can be found in Ref. [25]) on the local free volume was investigated. Similar materials have been studied previously [26] where the incorporation of the $m-\mathrm{CB}$ was found to result in a mechanical reinforcement of the material, increasing the equilibrium compression modulus $G^{\prime}$, and reducing the level of hysteresis in the cycled compression stress-strain analysis performed at $298 \mathrm{~K}$, as shown in Table II. The incorporation of $m$-CB $(0,1.8$ and $3.7 \mathrm{wt} \%)$ into the monomodal PDMS network did not change the glass transition temperature $\left(T_{\mathrm{g}} \approx 152 \mathrm{~K}\right)$, of the final composite, observed using both PALS and differential scanning calorimetry (DSC). Samples were studied across the temperature range $100-300 \mathrm{~K}$ in order to gain an understanding of how the nanoparticles affect the local free volume in both, the glassy and rubbery states. No significant change in the local free volume was observed between samples in the glassy state or above the so-called knee-temperature $\left(T_{\mathrm{k}} \approx 210 \mathrm{~K}\right)$, which occurs at a very similar temperature to the melting of the PDMS crystalline fraction (all of the samples studied were semicrystalline). Above $T_{\mathrm{k}}$ the PDMS structural relaxation occurs on the same time-scale as the $o$-Ps lifetime so the environment in which the $o-\mathrm{Ps}$ is localized is no longer constant during the lifetime of the $o-\operatorname{Ps}[27]$.

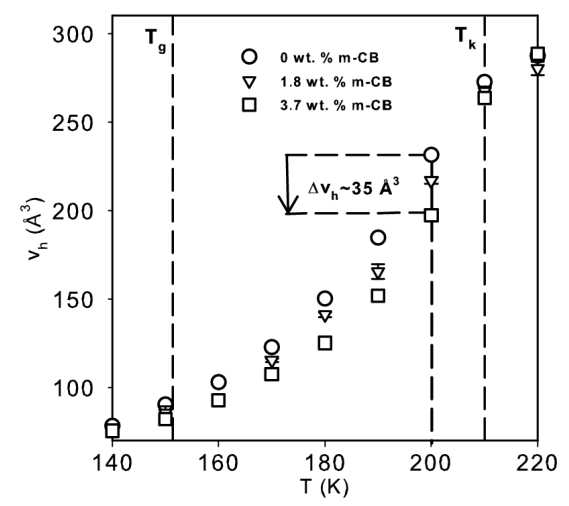

Fig. 4. Average hole volume $\left(v_{\mathrm{h}}\right)$ between $T_{\mathrm{g}}$ and $T_{\mathrm{k}}$ as measured by PALS for cross-linked monomodal PDMS networks with various loading levels of $m$-CB.

Figure 4 shows the average hole volume, calculated using the standard Tao-Eldrup model [8] (assuming spherical free volume element geometry), in the rubbery state between $T_{\mathrm{g}}$ and $T_{\mathrm{k}}$ (indicated). It is clear that the pres-

\section{TABLE II}

DMA results for cross-linked bimodal PDMS networks containing $m$-CB. Adapted from Ref. [26]. Copyright (2008) American Chemical Society.

\begin{tabular}{c|c|c}
\hline \hline wt\% $m$-CB & $G^{\prime}[\mathrm{MPa}]$ & Hysteresis width \\
\hline 0.0 & 0.19 & 5.5 \\
1.5 & 0.23 & 2.25 \\
3.7 & 0.32 & 0.33
\end{tabular}

ence of the $m$-CB is acting to reduce the average free volume hole size in this region, with a maximum decrease of $\approx 35 \mathrm{~m}^{3}(\approx 15 \%)$ at $200 \mathrm{~K}$ when $3.7 \mathrm{wt} \% \mathrm{~m}$-CB is present. Although this measurement was made at a much lower temperature, it is in good agreement with the findings from DMA on the bimodal PDMS networks [26].

A reduction in the average hole volume effectively reduces the space available to the polymer chains for reorganisation, hindering the chain mobility, reducing the viscous while enhancing the elastic behaviour of the material. This study is a preliminary investigation into exploring the correlation between free volume and mechanical reinforcement in polymer nanocomposites, more in depth work is currently under-way in Bristol to explore this relationship further, focussing on free volume measurements using PALS, mechanical properties using DMA and relaxation measurements using broadband dielectric spectroscopy (BDS) of other synthetic polymer composites.

\section{Final remarks}

By presenting a selection of examples, we have illustrated that although modern theories of phenomena such as the glass transition, self diffusion and the diffusion of guest molecules are more complicated than the original free volume concepts, the local free volume plays a fundamental role in governing many of the physical properties of polymeric matter. PALS has, therefore, proven to be an invaluable tool for the characterisation of a wide range of polymeric materials since it can directly probe the changes in thermodynamic state and molecular organisation at the sub-nanometer length scale.

\section{References}

[1] G. Dlubek, in: Polymer Physics: From Suspensions to Nanocomposites and Beyond, Eds. L.A. Utracki, A.M. Jamieson, John Wiley, Hoboken (NJ) 2011.

[2] F. Podczeck, B.E. Jones, Pharmaceutical Capsules, Pharmaceutical Press, London 2007.

[3] S. Kasapis, I.T. Norton, J.B. Ubbink, Modern Biopolymer Science, Elsevier, Amsterdam 2009.

[4] E. Donth, The Glass Transition: Relaxation Dynamics in Liquids and Disordered Materials, Springer, Berlin 2001.

[5] C.A. Kusmins, T.K. Kwei, in: Diffusion in Polymers, Eds. J. Crank, G.S. Park, Academic, London 1968. 
[6] J.S. Vrentas, J.L. Duda, J. Polym. Sci. Polym. Phys. Ed. 15, 403 (1977).

[7] M.H. Cohen, G.S. Grest, Phys. Rev. B 20, 1077 (1979).

[8] Positron and Positronium Chemistry, Eds. D.M. Schrader, Y.C. Jean, Elsevier, Amsterdam 1988.

[9] Y.C. Jean, P.E. Mallon, D.M. Schrader, Principles and Applications of Positron and Positronium Chemistry, World Sci., Singapore 2003.

[10] D. Kilburn, J. Claude, R. Mezzenga, G. Dlubek, A. Alam, J. Ubbink, J. Phys. Chem. B 108, 12436 (2004).

[11] D. Kilburn, J. Claude, T. Schweizer, A. Alam, J. Ubbink, Biomacromolecules 6, 864 (2005).

[12] S. Townrow, M. Roussenova, M. Giardiello, A. Alam, J. Ubbink, J. Phys. Chem. B 114, 1568 (2010).

[13] B.D. Freeman, Macromolecules 32, 375 (1999).

[14] H. Hayashi, A.P. Cote, H. Furukawa, M. O’Keeffe, O.M. Yaghi, Nat. Mater. 6, 501 (2007).

[15] Q. Song, S. Nataraj, M. Roussenova, J. Tan, D. Hughes, W. Li, P. Bourgoin, M.A. Alam, A. Cheetham, S. Al-Muhtaseb, E. Sivaniah, Energy Environ. Sci. 5, 8359 (2012).

[16] D.Q. Vu, W.J. Koros, S.J. Miller, J. Membr. Sci. 211, 311 (2003).

[17] V.P. Shantarovich, I.B. Kevdina, Y.P. Yampolskii, A.Y. Alentiev, Macromolecules 33, 7453 (2000).
[18] K.S. Park, Z. Ni, A.P. Cote, J.Y. Choi, R. Huang, F.J. Uribe-Romo, H.K. Chae, M. O'Keeffe, O.M. Yaghi, Proc. Natl. Acad. Sci. USA 103, 10186 (2006).

[19] P.J.A. Sobral, F.C. Menegalli, M.D. Hubinger, M.A. Roques, Food Hydrocolloids 15, 423 (2001).

[20] M. Roussenova, J. Enrione, P. Diaz-Calderon, A.J. Taylor, J. Ubbink, M.A. Alam, New J. Phys. 14, 035016 (2012).

[21] S. Townrow, D. Kilburn, A. Alam, J. Ubbink, J. Phys. Chem. B 111, 12643 (2007).

[22] M. Roussenova, M. Murith, A. Alam, J. Ubbink, Biomacromolecules 11, 3237 (2010).

[23] A.C. Balazs, T. Emrick, T.P. Russell, Science 314, 1107 (2006).

[24] J. Kalfus, J. Jancar, Compos. Sci. Technol. 68, 3444 (2008).

[25] D.J. Hughes, M.V. Roussenova, P. Beavis, A.C. Swain, M.A. Alam, J. Phys., Conf. Ser. 443, 012045 (2013).

[26] J.P. Lewicki, R.S. Maxwell, M. Patel, J.L. Herberg, A.C. Swain, J.J. Liggat, R.A. Pethrick, Macromolecules 41, 9179 (2008).

[27] G. Dlubek, U. De, J. Pionteck, N.Y. Arutyunov, M. Edelmann, R. Krause-Rehberg, Macromol. Chem. Phys. 206, 827 (2005). 